PROCEEDINGS OF THE

AMERICAN MATHEMATICAL SOCIETY

Volume 138, Number 7, July 2010, Pages 2413-2423

S 0002-9939(10)10290-1

Article electronically published on March 15, 2010

\title{
BAIRE CATEGORY AND THE WEAK BANG-BANG PROPERTY FOR CONTINUOUS DIFFERENTIAL INCLUSIONS
}

\author{
F. S. DE BLASI AND G. PIANIGIANI
}

(Communicated by Yingfei Yi)

\begin{abstract}
For continuous differential inclusions the classical bang-bang property is known to fail, yet a weak form of it is established here, in the case where the right hand side is a multifunction whose values are closed convex and bounded sets with nonempty interior contained in a reflexive and separable Banach space. Our approach is based on the Baire category method.
\end{abstract}

\section{INTRODUCTION}

The bang-bang property for differential inclusions has been studied by many authors from different points of view. For recent contributions see, among others, Papageorgiou [14], Tolstonogov [18, Donchev, Farkhi, Mordukhovich [10]. A comprehensive account on differential inclusions and additional references can be found in the monographs by Aubin and Cellina [1, Hu and Papageorgiou [12, Mordukhovich [13, and Tolstonogov [17. Usually in the investigation of the bang-bang property a crucial role is played by the assumption that the differential inclusion satisfies a globally Lipschitz condition. Recently, it has been shown that the bangbang property remains valid even under a locally Lipschitz condition 9, while it is known to be false under the mere assumption of continuity in view of an example of Plis [16. The aim of the present paper is to show that, under appropriate assumptions, a somewhat weaker form of the bang-bang property is valid for continuous differential inclusions (Theorem 1). Our method of approach is based on the Baire category as developed in [6]-[9]. To apply it we need some technical results, among which is a suitable infinite dimensional version of the classical Carathéodory theorem concerning compact convex sets in $\mathbb{R}^{n}$ (Proposition 4). For further details on the Baire method, see [4, 12, [15].

Let $(M, \rho)$ be a metric space. The interior and the closure of a set $X \subset M$ are denoted by $\operatorname{int} X$ and $\bar{X}$. For $a \in M$ and $X \subset M, X \neq \emptyset$, we set $d(a, X)=$ $\inf _{x \in X} \rho(a, X)$.

Throughout the paper $\mathbb{E}$ is a reflexive and separable real Banach space with norm $\|$.$\| and \mathcal{B}(\mathbb{E})($ resp. $\mathcal{C}(\mathbb{E}))$ is the space of all subsets of $\mathbb{E}$ which are closed convex bounded with nonempty interior (resp. closed convex bounded nonempty). The spaces $\mathcal{B}(\mathbb{E}), \mathcal{C}(\mathbb{E})$ are equipped with the Pompeiu-Hausdorff metric

$$
h(X, Y)=\max \left\{\sup _{x \in X} d(x, Y), \sup _{y \in Y} d(y, X)\right\} .
$$

Received by the editors September 8, 2009.

2010 Mathematics Subject Classification. Primary 34AXX. 
If $X \subset \mathbb{E}$, then coX denotes the convex hull of $X$. If $X \subset \mathbb{E}$ is convex, then ext $X$ denotes the set of all extreme points of $X$. For $X \subset \mathbb{E}, X \neq \emptyset$, we put $\|X\|=\sup \{\|x\| \mid x \in X\}$. Moreover $B=\{x \in \mathbb{E} \mid\|x\| \leq 1\}$.

As usual $C(I, \mathbb{E})$, where $I \subset \mathbb{R}$ is a compact interval, denotes the space of all continuous functions $x: I \rightarrow \mathbb{E}$ with norm $\|x\|_{I}=\max \{t \in I \mid\|x(t)\|\}$.

Set $I=\left[t_{o}, t_{1}\right], t_{0}<t_{1}$. A multifunction $F: I \times \mathbb{E} \rightarrow \mathcal{C}(\mathbb{E})$ is said to satisfy assumption $(H)$ if :

(j) $F$ is continuous on $I \times \mathbb{E}$,

(jj) $F$ is bounded, i.e. $\|F(t, x)\|<M$ for every $(t, x) \in I \times \mathbb{E}, M$ a positive constant.

For $F$ satisfying $(H)$ and $a \in \mathbb{E}$, consider the following Cauchy problems:

$\begin{array}{ll}\left(C_{F, a}\right) & \dot{x}(t) \in F(t, x(t)), \quad x\left(t_{0}\right)=a, \\ \left(C_{\text {int } F, a}\right) & \dot{x}(t) \in \operatorname{int} F(t, x(t)), \quad x\left(t_{0}\right)=a, \\ \left(C_{\operatorname{ext} F, a}\right) & \dot{x}(t) \in \operatorname{ext} F(t, x(t)), \quad x\left(t_{0}\right)=a .\end{array}$

A function $x: I \rightarrow \mathbb{E}$ is said to be a solution of the Cauchy problem $\left(C_{F, a}\right)$ (resp. $\left.\left(C_{\text {int } F, a}\right),\left(C_{\operatorname{ext} F, a}\right)\right)$ if $x$ is Lipschitzian on $I$, with $x\left(t_{0}\right)=a$, and satisfies $\left(C_{F, a}\right)$ (resp. $\left.\left(C_{\text {int } F, a}\right),\left(C_{\text {ext } F, a}\right)\right), t \in I$ a.e.

For $F$ satisfying $(H)$ and $a \in \mathbb{E}$ set:

$$
\begin{aligned}
\mathcal{M}_{F, a} & =\left\{x: I \rightarrow \mathbb{E} \mid x \text { is a solution of }\left(C_{F, a}\right)\right\}, \\
\mathcal{M}_{\operatorname{int} F, a} & =\left\{x: I \rightarrow \mathbb{E} \mid x \text { is a solution of }\left(C_{\operatorname{int} F, a}\right)\right\} \\
\mathcal{M}_{\operatorname{ext} F, a} & =\left\{x: I \rightarrow \mathbb{E} \mid x \text { is a solution of }\left(C_{\operatorname{ext} F, a}\right)\right\} .
\end{aligned}
$$

The space $\mathcal{M}_{F, a}$ is equipped with the metric induced by the norm of $C(I, E)$, i.e. the metric of uniform convergence.

It is evident that $\mathcal{M}_{\text {ext } F, a}$ and $\mathcal{M}_{\text {int } F, a}$ are contained in $\mathcal{M}_{F, a}$. Furthermore $\mathcal{M}_{F, a}$ can be empty if $\mathbb{E}$ is infinite dimensional and $F$ is merely continuous.

Under the assumption that $F: I \times \mathbb{E} \rightarrow \mathcal{C}(\mathbb{E})$ is continuous, locally Lipschitzian in the $x$-variable and bounded, the set $\mathcal{M}_{F, a}$ is nonempty and moreover the following bang-bang property holds (see [9]):

$$
\overline{\mathcal{M}}_{\text {ext } F, a}=\mathcal{M}_{F, a},
$$

where the closure is in $C(I, \mathbb{E})$. Whenever $F$ is only continuous, then the bang-bang property is no longer valid and one has

$$
\overline{\mathcal{M}}_{\text {ext } F, a} \subset \mathcal{M}_{F, a},
$$

where the inclusion can be strict, in view of the Plis example [16].

The aim of this paper is to show that if $F: I \times \mathbb{E} \rightarrow \mathcal{B}(\mathbb{E})$ is continuous and bounded, then $\mathcal{M}_{\text {int } F, a} \neq \emptyset$ and the following weak form of the bang-bang property holds:

$$
\overline{\mathcal{M}}_{\text {ext } F, a} \supset \mathcal{M}_{\text {int } F, a} .
$$

Remark 1. If $F$ takes on values in $\mathcal{C}(\mathbb{E})$ and $\mathbb{E}$ is infinite dimensional, then one can have $\mathcal{M}_{\text {ext } F, a}=\mathcal{M}_{F, a}=\emptyset$, by virtue of Godunov's theorem [11].

Remark 2. The inclusion (1.1) can be strict, as is shown by an example presented in Section 3. 
For any set $J \subset I$ we denote by $|J|$ and $\chi_{J}$, respectively, the Lebesgue measure and the characteristic function of $J$.

By a regular partition of the interval $I=\left[t_{0}, t_{1}\right]$ we mean a finite or denumerable infinite family $\mathcal{P}=\left\{I_{i}\right\}$ of pairwise disjoint nondegenerate intervals $I_{i} \subset I$ such that, setting $N_{0}=I \backslash \bigcup_{i} I_{i}$, one has $\left|N_{0}\right|=0$.

Definition 1. A map $u: I \rightarrow \mathbb{E}$ is said to be piecewise constant if $u$ is given by

$$
u(t)=\sum_{i} u_{i} \chi_{I_{i}}(t)+u_{0}(t) \chi_{N_{0}}(t), \quad t \in I,
$$

where $\left\{I_{i}\right\}$ is a regular partition of $I,\left\{u_{i}\right\} \subset \mathbb{E}$ is a bounded sequence, and $u_{0}(t) \in \mathbb{E}$ for every $t \in N_{0}=I \backslash \bigcup_{i} I_{i}$.

Definition 2. A solution $x \in \mathcal{M}_{F, a}$ is said to be regular if there exist a regular partition $\mathcal{P}=\left\{I_{i}\right\}$ of $I$ and corresponding sequences $\left\{u_{i}\right\} \subset \mathbb{E}$ and $\left\{\sigma_{i}\right\} \subset(0,+\infty)$ such that, denoting by $u: I \rightarrow \mathbb{E}$ a piecewise constant map given by (1.2), one has:

$$
x(t)=a+\int_{t_{0}}^{t} u(s) d s \text { for each } t \in I,
$$

$$
\dot{x}(t)+\sigma_{i} B=u_{i}+\sigma_{i} B \subset F(t, x(t)) \text { for each } t \in \operatorname{int} I_{i} \text { and } I_{i} \in \mathcal{P} .
$$

Set

and define

$$
\mathcal{M}_{F, a}^{0}=\left\{x: I \rightarrow \mathbb{E} \mid x \text { is a regular solution of }\left(C_{\text {int } F, a}\right)\right\},
$$

$$
\mathcal{M}=\overline{\mathcal{M}}_{F, a}^{0}
$$

where the closure is in $C(I, \mathbb{E})$. The space $\mathcal{M}$ is equipped with the metric induced by the norm of $C(I, \mathbb{E})$.

The Choquet function, which we now introduce, plays a crucial role in the proof of our main result.

Denote by $\mathbb{E}^{*}$ the topological dual of $\mathbb{E}$. Let $\left\{l_{n}\right\},\left\|l_{n}\right\|=1$, be a sequence dense in the unit sphere of $\mathbb{E}^{*}$. Let $F$ satisfy assumption $(H)$. Following Choquet [5], Vol. II, Ch. 6 , we define $\varphi_{F}: I \times \mathbb{E} \times \mathbb{E} \rightarrow[0,+\infty]$ by

$$
\varphi(t, x, v)=\left\{\begin{array}{l}
\sum_{n=1}^{\infty} \frac{\left(l_{n}(v)\right)^{2}}{2^{n}}, \quad v \in F(t, x), \\
+\infty, \quad v \in \mathbb{E} \backslash F(t, x) .
\end{array}\right.
$$

Let $\mathcal{A}$ be the set of all continuous affine functions $a: \mathbb{E} \rightarrow \mathbb{R}$. Let $\bar{\varphi}_{F}: I \times \mathbb{E} \times \mathbb{E} \rightarrow$ $[-\infty,+\infty)$ be given by

$$
\bar{\varphi}_{F}(t, x, v)=\inf \left\{a(v) \mid a \in \mathcal{A} \text { and } a(z)>\varphi_{F}(t, x, z) \text { for every } z \in F(t, x)\right\} .
$$

We define $d_{F}: I \times \mathbb{E} \times \mathbb{E} \rightarrow[-\infty,+\infty)$ by

$$
d_{F}(t, x, v)=\bar{\varphi}_{F}(t, x, v)-\varphi_{F}(t, x, v) .
$$

In the next proposition we review some properties of $d_{F}$, the Choquet function associated to $F$ (see Choquet [5], Castaing and Valadier [2]).

Proposition 1. Let $F: I \times \mathbb{E} \rightarrow \mathcal{B}(\mathbb{E})$ satisfy $(H)$. Then:

(i) for each $(t, x) \in I \times \mathbb{E}$ and $v \in F(t, x)$ we have $0 \leq d_{F}(t, x, v) \leq M^{2}$. Moreover $d_{F}(t, x, v)=0$ if and only if $v \in \operatorname{ext} F(t, x)$;

(ii) for each $(t, x) \in I \times \mathbb{E}$, the function $d_{F}(t, x,$.$) is concave on \mathbb{E}$ and strictly concave on the set $F(t, x)$;

(iii) $d_{F}$ is upper semicontinuous on $I \times \mathbb{E} \times \mathbb{E}$; 
(iv) for each $x \in \mathcal{M}_{F, a}$, the function $t \rightarrow d_{F}(t, x(t), \dot{x}(t))$ is nonnegative, bounded and integrable on $I$;

(v) if $\left\{x_{n}\right\} \subset \mathcal{M}_{F, a}$ converges uniformly to $x$, then

$$
\limsup _{n \rightarrow \infty} \int_{I} d_{F}\left(t, x_{n}(t), \dot{x}_{n}(t)\right) d t \leq \int_{I} d_{F}(t, x(t), \dot{x}(t)) d t .
$$

\section{Auxiliary Results}

In this section we prove some results which will be useful in what follows.

Proposition 2. Let $F: I \times \mathbb{E} \rightarrow \mathcal{B}(\mathbb{E})$ satisfy $(H)$. Let $x \in \mathcal{M}_{\operatorname{int} F, a}$ and $\varepsilon>0$. Then there exists a regular solution $y: I \rightarrow \mathbb{E}$ of the Cauchy problem $\left(C_{\operatorname{int} F, a}\right)$ such that $\|y-x\|_{I}<\varepsilon$.

Proof. Let $x \in \mathcal{M}_{\text {int } F, a}$ and $\varepsilon>0$ be given. Set

$$
J=\left\{t \in\left(t_{0}, t_{1}\right) \mid \dot{x}(t) \text { exists and } \dot{x}(t) \in \operatorname{int} F(t, x(t))\right\} .
$$

Let $\tau \in J$ be arbitrary. Hence for some $\sigma>0$ we have

$$
\dot{x}(\tau)+3 \sigma B \subset F(\tau, x(\tau)) .
$$

Since $F$ is continuous, there exists $\delta_{\tau}>0$, with $\left[\tau, \tau+\delta_{\tau}\right] \subset\left(t_{0}, t_{1}\right)$, such that

$0<h<\delta_{\tau},|t-\tau|<\delta_{\tau}, t \in I,\|z-x(\tau)\|<\delta_{\tau} \Rightarrow \frac{x(\tau+h)-x(\tau)}{h}+\sigma B \subset F(t, z)$.

For any $\delta$ with

$$
0<\delta<\min \left\{\frac{\delta_{\tau}}{M+1}, \frac{\varepsilon}{2 M+1}\right\},
$$

set $I_{\tau, \delta}=[\tau, \tau+\delta]$ and define $y_{\tau, \delta}: I_{\tau, \delta} \rightarrow \mathbb{E}$ by

$$
y_{\tau, \delta}(t)=x(\tau)+\frac{x(\tau+\delta)-x(\tau)}{\delta}(t-\tau), \quad t \in I_{\tau, \delta} .
$$

Claim 1. $y_{\tau, \delta}: I_{\tau, \delta} \rightarrow \mathbb{E}$ is a regular solution of the following boundary value problem:

$\left(B_{\tau, \delta}\right) \quad \dot{y}(t)=\operatorname{int} F(t, y(t)), \quad y(\tau)=x(\tau), \quad y(\tau+\delta)=x(\tau+\delta)$.

Evidently $y(\tau)=x(\tau)$ and $y(\tau+\delta)=x(\tau+\delta)$. Moreover for $t \in I_{\tau, \delta}$ we have

$$
\left\|y_{\tau, \delta}(t)-x(\tau)\right\|=\frac{\|x(\tau+\delta)-x(\tau)\|}{\delta}(t-\tau)<\delta_{\tau},
$$

because $\|x(\tau+\delta)-x(\tau)\|<M \delta$ and $t-\tau \leq \delta<\delta_{\tau} /(M+1)$. From (2.1) (with $h=\delta)$, in view of $(2.2)$ it follows that

$$
\dot{y}_{\tau, \delta}(t)+\sigma B=\frac{x(\tau+\delta)-x(\tau)}{\delta}+\sigma B \subset F\left(t, y_{\tau, \delta}(t)\right), \quad t \in(\tau, \tau+\delta),
$$

and hence Claim 1 holds.

Now the family

$$
\mathcal{F}=\left\{I_{\tau, \delta}, \tau \in J, 0<\delta<\delta_{\tau}\right\}
$$

of closed intervals $I_{\tau, \delta}$ covers $J$ in the sense of Vitali. Hence there exists a finite or denumerable infinite family $\mathcal{F}_{0}=\left\{I_{\tau_{j}, \delta_{j}}\right\} \subset \mathcal{F}$ of pairwise disjoint closed intervals 
$I_{\tau_{j}, \delta_{j}} \in \mathcal{F}$ such that $J \backslash \bigcup_{j} I_{\tau_{j}, \delta_{j}}$ has measure zero. As $I \backslash J$ has measure zero it follows that $N_{0}=I \backslash \bigcup_{j} I_{\tau_{j}, \delta_{j}}$ has measure zero. Evidently

$$
I=\bigcup_{j} I_{\tau_{j}, \delta_{j}} \cup N_{0},
$$

and thus $\mathcal{F}_{0}$ is a regular partition of $I$. Now define $u: I \rightarrow \mathbb{E}$ and $y: I \rightarrow \mathbb{E}$ as follows:

$$
\begin{aligned}
& u(t)=\sum_{j} \dot{y}_{\tau_{j}, \delta_{j}}(t) \chi_{I_{\tau_{j}, \delta_{j}}}(t), \quad t \in I, \\
& y(t)=a+\int_{t_{0}}^{t} u(s) d s, \quad t \in I .
\end{aligned}
$$

Claim 2. $y: I \rightarrow \mathbb{E}$ is a regular solution of the Cauchy problem $\left(C_{\text {int } F, a}\right)$ satisfying $\|y-x\|_{I}<\varepsilon$.

Since the functions $y_{\tau_{j}, \delta_{j}}$ and $x$ agree at $\tau_{j}$ and $\tau_{j}+\delta_{j}$, the end points of $I_{\tau_{j}, \delta_{j}}$, then in view of the definition of $y$ it is easy to show that $y(t)=y_{\tau_{j}, \delta_{j}}(t)$ for each $t \in I_{\tau_{j}, \delta_{j}}$ and $I_{\tau_{j}, \delta_{j}} \in \mathcal{F}_{0}$. By virtue of Claim 1 and (2.3) it follows that $y$ is a regular solution of the Cauchy problem $\left(C_{\operatorname{int} F, a}\right)$. Furthermore for any $I_{\tau_{j}, \delta_{j}} \in \mathcal{F}_{0}$ and all $t \in I_{\tau_{j}, \delta_{j}}$ we have

$$
\|y(t)-x(t)\| \leq\left\|y(t)-y\left(\tau_{j}\right)\right\|+\left\|x\left(\tau_{j}\right)-x(t)\right\|<2 M \delta_{j} .
$$

As $\delta_{j}<\varepsilon /(2 M+1)$, in view of (2.3) it follows that $\|y-x\|_{I}<\varepsilon$. Therefore Claim 2 holds. This completes the proof.

Proposition 3. Let $F: I \times \mathbb{E} \rightarrow \mathcal{B}(\mathbb{E})$ satisfy $(H)$. Then $\mathcal{M}$ is a nonempty complete metric space (under the induced metric of $C(I, \mathbb{E})$ ) and $\mathcal{M} \subset \mathcal{M}_{F, a}$.

Proof. Since the multifunction int $F$ admits locally Lipschitzian selections, we have $\mathcal{M}_{\text {int } F, a} \neq \emptyset$ and thus $\mathcal{M}_{F, a}^{0} \neq \emptyset$, by virtue of Proposition 2. Hence $\mathcal{M} \neq \emptyset$. Evidently $\mathcal{M}$ is complete for $C(I, \mathbb{E})$ is so. As $F$ is a continuous and bounded multifunction with closed convex values contained in $\mathbb{E}$, a reflexive Banach space, the uniform limit of solutions is also a solution, and hence $\mathcal{M} \subset \mathcal{M}_{F, a}$.

Proposition 4. Let $F: I \times \mathbb{E} \rightarrow \mathcal{B}(\mathbb{E})$ satisfy $(H)$. Let $(t, x) \in I \times \mathbb{E}$ and let $u \in \operatorname{int} F(t, x)$ and $\alpha>0$ be given. Then for some $n \in \mathbb{N}$ there exist points $a_{i} \in \operatorname{int} F(t, x)$, with $d_{F}\left(t, x, a_{i}\right)<\alpha, i=1, \ldots, n$, and numbers $\lambda_{i}>0$, with $\lambda_{1}+\cdots+\lambda_{n}=1$, such that

$$
\sum_{i=1}^{n} \lambda_{i} a_{i}=u
$$

Proof. Fix $\theta>0$ so that $u+\theta B \subset F(t, x)$. For some $p \in \mathbb{N}$ there exist points $e_{i} \in \operatorname{ext} F(t, x)$ and numbers $\mu_{i}>0$ with $\mu_{1}+\cdots+\mu_{p}=1$, such that, setting $c=\mu_{1} e_{1}+\cdots+\mu_{p} e_{p}$, one has

$$
0<\|c-u\|<\frac{\theta}{2} .
$$

By Proposition $1, d_{F}\left(t, x, e_{i}\right)=0, i=1, \ldots, p$, and thus sufficiently close to each $e_{i}$ there exists a point $a_{i} \in \operatorname{int} F(t, x)$ such that

$$
d_{F}\left(t, x, a_{i}\right)<\frac{\alpha}{2}, i=1, \ldots, p \quad \text { and } \quad 0<r=\|a-u\|<\frac{\theta}{2},
$$


where $a=\mu_{1} a_{1}+\cdots+\mu_{p} a_{p}$. By virtue of Proposition 1 and (2.4) there exists an $\varepsilon>0$ such that each ball $B_{i}=a_{i}+\varepsilon B, i=1, \ldots, p$, is contained in $F(t, x)$ and moreover,

$$
d_{F}(t, x, v)<\alpha \quad \text { for every } v \in B_{i}, i=1, \ldots, p .
$$

It is evident that

$$
\sum_{i=1}^{p} \mu_{i} B_{i}=B_{\varepsilon}(a), \quad \text { where } B_{\varepsilon}(a)=a+\varepsilon B .
$$

Let $b \in \operatorname{int} F(t, x)$ be such that $u=\frac{a+b}{2}$. As above, for some $q \in \mathbb{N}$ there exist points $a_{i}^{\prime} \in \operatorname{int} F(t, x)$, with $d_{F}\left(t, x, a_{i}^{\prime}\right)<\alpha, i=1, \ldots, q$, and numbers $\nu_{i}>0$, with $\nu_{1}+\cdots+\nu_{q}=1$, such that setting

$$
b^{\prime}=\sum_{i=1}^{q} \nu_{i} a_{i}^{\prime}
$$

one has $\left\|b^{\prime}-b\right\|<\frac{\varepsilon}{4}$. As $u+\frac{\varepsilon}{2} B \subset \operatorname{co}\left\{b, B_{\varepsilon}(a)\right\}$ and $h\left(\operatorname{co}\left\{b, B_{\varepsilon}(a)\right\}, \operatorname{co}\left\{b^{\prime}, B_{\varepsilon}(a)\right\}\right) \leq$ $\left\|b^{\prime}-b\right\|<\frac{\varepsilon}{4}$, it follows that $u+\frac{\varepsilon}{2} B \subset \operatorname{co}\left\{b^{\prime}, B_{\varepsilon}(a)\right\}+\frac{\varepsilon}{4} B$, which implies $u+\frac{\varepsilon}{4} B \subset$ $\operatorname{co}\left\{b^{\prime}, B_{\varepsilon}(a)\right\}$. Hence there exist $d \in B_{\varepsilon}(a)$ and $t \in[0,1]$ so that

$$
u=t b^{\prime}+(1-t) d .
$$

As $d \in B_{\varepsilon}(a)$, by virtue of (2.6) and (2.5) there exist points $d_{i}^{\prime} \in B_{i}$, with $d_{F}\left(t, x, d_{i}^{\prime}\right)$ $<\alpha, i=1, \ldots, p$, such that

$$
d=\sum_{i=1}^{p} \mu_{i} d_{i}^{\prime}
$$

From (2.8), in view of (2.7) and (2.9), it follows that

$$
u=t \sum_{i=1}^{q} \nu_{i} a_{i}^{\prime}+(1-t) \sum_{i=1}^{p} \mu_{i} d_{i}^{\prime} .
$$

This completes the proof.

\section{THE WEAK BANG-BANG PROPERTY}

In this section we shall prove the weak bang-bang property. To this end, for $F: I \times \mathbb{E} \rightarrow \mathcal{B}(\mathbb{E})$ satisfying $(H)$ and $\alpha>0$, we set

$$
\mathcal{N}_{\alpha}=\left\{x \in \mathcal{M}: \int_{I} d_{F}(t, x(t), \dot{x}(t)) d t<\alpha|I|\right\} .
$$

Proposition 5. $\mathcal{N}_{\alpha}$ is dense in $\mathcal{M}$.

Proof. It suffices to show that given $x \in \mathcal{M}_{F, a}^{0}$ and $\varepsilon>0$ there exists $y \in \mathcal{N}_{\alpha}$ such that $\|y-x\|_{I} \leq \varepsilon$.

By hypothesis $x$ is a regular solution of $\left(C_{\operatorname{int} F, a}\right)$ and thus, with the notation of Definition 2, for some piecewise constant map $u: I \rightarrow \mathbb{E}$, given by (1.2), we have:

$$
\begin{gathered}
x(t)=a+\int_{t_{0}}^{t} u(s) d s, \quad t \in I, \\
\dot{x}(t)+\sigma_{i} B=u_{i}+\sigma_{i} B \subset F(t, x(t)), \quad t \in \operatorname{int} I_{i}, I_{i} \in \mathcal{P} .
\end{gathered}
$$


Consider an interval $I_{i} \in \mathcal{P}$ with end points $\alpha_{i}<\beta_{i}$ and let $\tau \in\left(\alpha_{i}, \beta_{i}\right)$ be arbitrary. Evidently

$$
u_{i}+\sigma_{i} B \subset F(\tau, x(\tau)),
$$

and thus by Proposition 4, for some $n \in \mathbb{N}$ there exist points $e_{k} \in \operatorname{int} F(\tau, x(\tau))$, with $d_{F}(\tau, x(\tau), \dot{x}(\tau))<\alpha, k=1, \ldots, n$, and corresponding numbers $\lambda_{k}>0$, with $\lambda_{1}+\cdots+\lambda_{n}=1$, such that

$$
u_{i}=\sum_{k=1}^{n} \lambda_{k} e_{k}
$$

Clearly for some $\gamma_{\tau}>0$,

$$
\bigcup_{k=1}^{n}\left(e_{k}+\gamma_{\tau} B\right) \subset F(\tau, x(\tau))
$$

Since $F$ is continuous and satisfies (3.2) there exists $\rho_{0}$, with

$$
0<\rho_{0}<\min \left\{\varepsilon, \tau-\alpha_{i}, \beta_{i}-\tau\right\},
$$

such that

$$
t \in\left[\tau-\rho_{0}, \tau+\rho_{0}\right],\|y-x(\tau)\|<\rho_{0} \Rightarrow \bigcup_{k=1}^{n}\left(e_{k}+\frac{\gamma_{\tau}}{2} B\right) \subset F(t, y) .
$$

Furthermore as $d_{F}$ is upper semicontinuous at $\left(\tau, x(\tau), e_{k}\right)$ and $d_{F}\left(\tau, x(\tau), e_{k}\right)<\alpha$ there exists a $\rho$, with $0<\rho<\min \left\{\rho_{0}, \gamma_{\tau} / 2\right\}$, such that for $k=1, \ldots, n$ we have

$$
t \in[\tau-\rho, \tau+\rho],\|y-x(\tau)\|<\rho,\left\|v-e_{k}\right\|<\rho \quad \Rightarrow \quad d_{F}(t, y, v)<\alpha .
$$

Let $\delta_{\tau}$ satisfy

$$
0<\delta_{\tau}<\frac{\rho}{4 M+1}
$$

and, for $0<\delta<\delta_{\tau}$, set

$$
I_{\tau, \delta}=[\tau-\delta, \tau+\delta]
$$

Let $\left\{J_{\tau, \delta}^{k}\right\}_{k=1}^{n}$ be a partition of $I_{\tau, \delta}$ into $n$ pairwise disjoint nondegenerate subintervals $J_{\tau, \delta}^{k}$ of length

$$
\left|J_{\tau, \delta}^{k}\right|=\lambda_{k}\left|I_{\tau, \delta}\right|, k=1, \ldots, n .
$$

Now define $u_{\tau, \delta}: I \rightarrow \mathbb{E}$ and $y_{\tau, \delta}: I_{\tau, \delta} \rightarrow \mathbb{E}$ as follows:

$$
\begin{aligned}
& u_{\tau, \delta}(t)=\sum_{k=1}^{n} e_{k} \chi_{J_{\tau, \delta}^{k}}(t), t \in I_{\tau, \delta}, \\
& y_{\tau, \delta}(t)=x(\tau-\delta)+\int_{\tau-\delta}^{t} u_{\tau, \delta}(s) d s, t \in I_{\tau, \delta} .
\end{aligned}
$$


We have

$$
\begin{gathered}
\dot{y}_{\tau, \delta}(t)+\frac{\gamma_{\tau}}{2} B \subset F\left(t, y_{\tau, \delta}(t)\right), \quad t \in \bigcup_{k=1}^{n} \operatorname{int} J_{\tau, \delta}^{k}, \\
y_{\tau, \delta}(\tau \pm \delta)=x(\tau \pm \delta), \\
\left\|y_{\tau, \delta}(t)-x(t)\right\|<\varepsilon, t \in I_{\tau, \delta}, \\
d_{F}\left(t, y_{\tau, \delta}(t), \dot{y}_{\tau, \delta}(t)\right)<\alpha, \quad t \in \bigcup_{k=1}^{n} \operatorname{int} J_{\tau, \delta}^{k} .
\end{gathered}
$$

(j) Let $t \in \operatorname{int} J_{\tau, \delta}^{k}$, where $1 \leq k \leq n$. Clearly

$$
t \in[\tau-\rho, \tau+\rho]
$$

since $|t-\tau| \leq \delta<\delta_{\tau}<\rho$ by (3.6). Moreover,

$$
\begin{aligned}
& \left\|y_{\tau, \delta}(t)-x(\tau)\right\|=\left\|x(\tau-\delta)+\int_{\tau-\delta}^{t} u_{\tau, \delta}(s) d s-a-\int_{t_{0}}^{\tau} u(s) d s\right\| \\
& \quad=\left\|\int_{\tau-\delta}^{t} u_{\tau, \delta}(s) d s-\int_{\tau-\delta}^{\tau} u(s) d s\right\| \\
& \quad \leq \int_{J_{\tau, \delta}^{k}}\left(\left\|u_{\tau, \delta}(s)\right\|+\|u(s)\|\right) d s \leq 4 M \delta_{\tau},
\end{aligned}
$$

for $\left|J_{\tau, \delta}^{k}\right| \leq\left|I_{\tau, \delta}\right|=2 \delta<2 \delta_{\tau}$ and $u_{\tau, \delta}$ and $u$ are bounded by $M$. Since $\delta_{\tau}<$ $\rho /(4 M+1)$ by $(3.6)$, it follows that

$$
\left\|y_{\tau, \delta}(t)-x(\tau)\right\|<\rho .
$$

From (3.4), in view of (3.10) and (3.11), as $\rho<\rho_{0}$ and $\dot{y}_{\tau, \delta}(t)=e_{k}$, one has

$$
\dot{y}_{\tau, \delta}(t)+\frac{\gamma_{\tau}}{2} B \subset F\left(t, y_{\tau, \delta}(t)\right)
$$

and thus $(\mathrm{j})$ holds

(jj) From (3.9), in view of (3.8), (3.7) and (3.1) we have:

$$
\begin{aligned}
& y_{\tau, \delta}(\tau+\delta)=x(\tau-\delta)+\int_{\tau-\delta}^{\tau+\delta}\left(\sum_{k=1}^{n} e_{k} \chi_{J_{\tau, \delta}^{k}}(s)\right) d s \\
& =x(\tau-\delta)+\sum_{k=1}^{n} e_{k}\left|J_{\tau, \delta}^{k}\right|=x(\tau-\delta)+\sum_{k=1}^{n} \lambda_{k} e_{k}\left|I_{\tau, \delta}\right| \\
& =x(\tau-\delta)+u_{i}\left|I_{\tau, \delta}\right|=x(\tau-\delta)+\int_{\tau-\delta}^{\tau+\delta} u(s) d s=x(\tau+\delta) .
\end{aligned}
$$

Clearly $y_{\tau, \delta}(\tau-\delta)=x(\tau-\delta)$, and thus (jj) holds.

(jjj) For any $t \in I_{\tau, \delta}$ we have:

$$
\begin{aligned}
& \left\|y_{\tau, \delta}(t)-x(t)\right\|=\left\|x(\tau-\delta)+\int_{\tau-\delta}^{t} u_{\tau, \delta}(s) d s-a-\int_{t_{0}}^{t} u(s) d s\right\| \\
& =\left\|\int_{\tau-\delta}^{t} u_{\tau, \delta}(s) d s-\int_{\tau-\delta}^{t} u(s) d s\right\| \leq \int_{I_{\tau, \delta}}\left(\left\|u_{\tau, \delta}(s)\right\|+\|u(s)\|\right) d s \leq 4 M \delta .
\end{aligned}
$$

From the latter, (jjj) follows at once since $\delta<\delta_{\tau}<\rho /(4 M+1)$ by (3.6), and $\rho<\rho_{0}<\varepsilon$ by $(3.3)$. 
(jv) Let $t \in \operatorname{int} J_{\tau, \delta}^{k}$ be arbitrary, where $1 \leq k \leq n$. From (3.5), in view of (3.10) and $(3.11)$, as $\dot{y}_{\tau, \delta}(t)=e_{k} \in F\left(t, y_{\tau, \delta}(t)\right)$, one has $d_{F}\left(t, y_{\tau, \delta}(t), \dot{y}_{\tau, \delta}(t)\right)<\alpha$, and thus (jv) holds.

It is evident that the family

$$
\mathcal{F}=\left\{I_{\tau, \delta} \mid \tau \in \bigcup_{i} \operatorname{int} I_{i}, 0<\delta<\delta_{\tau}\right\}
$$

of closed intervals $I_{\tau, \delta} \subset I$ covers the set $\bigcup_{i} \operatorname{int} I_{i}$ in the sense of Vitali. Hence there is a finite or denumerable infinite family $\mathcal{F}_{0}=\left\{I_{\tau_{j}, \delta_{j}}\right\} \subset \mathcal{F}$ of pairwise disjoint closed intervals $I_{\tau_{j}, \delta_{j}}$ such that

$$
I=\left(\bigcup_{j} I_{\tau_{j}, \delta_{j}}\right) \cup N_{0}
$$

where $N_{0}=I \backslash \bigcup_{j} I_{\tau_{j}, \delta_{j}}$ has measure zero. Now define $v: I \rightarrow \mathbb{E}$ and $y: I \rightarrow \mathbb{E}$ as follows:

$$
\begin{aligned}
& v(t)=\sum_{j} u_{\tau_{j}, \delta_{j}}(t) \chi_{I_{\tau_{j}, \delta_{j}}}(t), \quad t \in I, \\
& y(t)=a+\int_{t_{0}}^{t} v(s) d s, \quad t \in I .
\end{aligned}
$$

Since by (jj) $y_{\tau_{j}, \delta_{j}}\left(\tau_{j} \pm \delta_{j}\right)=x\left(\tau_{j} \pm \delta_{j}\right)$, it is easy to see that for each $I_{\tau_{j}, \delta_{j}} \in \mathcal{F}_{0}$ we have

$$
y(t)=y_{\tau_{j}, \delta_{j}}(t) \quad \text { for every } t \in I_{\tau_{j}, \delta_{j}} .
$$

In view of (3.13), (3.12) and (j) it follows that $y: I \rightarrow \mathbb{E}$ is a regular solution of the Cauchy problem $\left(C_{\operatorname{int} F, a}\right)$; hence $y \in \mathcal{M}_{\text {int } F, a}^{0}$ and so a fortiori $y \in \mathcal{M}$. Furthermore, by virtue of (3.13), (3.12) and (jv),

$$
\int_{I} d_{F}(t, y(t), \dot{y}(t)) d t<\alpha|I|
$$

and hence $y \in \mathcal{N}_{\alpha}$. Finally from (3.13), (3.12) and (jjj) it follows that $\|y-x\|_{I} \leq \varepsilon$. This completes the proof.

Proposition 6. $\mathcal{N}_{\alpha}$ is open in $\mathcal{M}$.

Proof. Let $\left\{x_{n}\right\} \subset \mathcal{M} \backslash \mathcal{N}_{\alpha}$ be a sequence which converges uniformly to $x \in \mathcal{M}$. Then, by Proposition 1,

$$
\int_{I} d_{F}(t, x(t), \dot{x}(t)) d t \geq \limsup _{n \rightarrow \infty} \int_{I} d_{F}\left(t, x_{n}(t), \dot{x}_{n}(t)\right) d t \geq \alpha|I| .
$$

Thus $x \in \mathcal{M} \backslash \mathcal{N}_{\alpha}$, completing the proof.

We are now ready to prove the following weak form of the bang-bang property.

Theorem 1. Let $F: I \times \mathbb{E} \rightarrow \mathcal{B}(\mathbb{E})$ satisfy assumption $(H)$. Then $\mathcal{M}_{\text {int } F, a} \neq \emptyset$ and

$$
\overline{\mathcal{M}}_{\text {ext } F, a} \supset \mathcal{M}_{\text {int } F, a} .
$$

Proof. Under our assumptions, $\mathcal{M}_{\operatorname{int} F, a} \neq \emptyset$. To prove (3.14) set

$$
\mathcal{M}^{*}=\bigcap_{n \in \mathbb{N}} \mathcal{N}_{1 / n} .
$$


By virtue of Propositions 5 and 6 each $\mathcal{N}_{1 / n}$ is open and dense in $\mathcal{M}$, which is a complete metric space, by Proposition 3. Consequently $\mathcal{M}^{*}$ is dense in $\mathcal{M}$.

Let $x \in \mathcal{M}^{*}$. Then for every $n \in \mathbb{N}$,

$$
\int_{I} d_{F}(t, x(t), \dot{x}(t)) d t<(1 / n)|I|,
$$

and thus, by Proposition $1, d_{F}(t, y(t), \dot{y}(t))=0, t \in I$ a.e., which implies that $x \in \mathcal{M}_{\text {ext } F, a}$. Therefore $\mathcal{M}_{\text {ext } F, a} \supset \mathcal{M}^{*}$ and hence,

$$
\overline{\mathcal{M}}_{\operatorname{ext} F, a} \supset \mathcal{M} \text {. }
$$

Since, by Proposition 2, $\mathcal{M}=\overline{\mathcal{M}}_{F, a}^{0} \supset \mathcal{M}_{\text {int } F, a}$, it follows that $\overline{\mathcal{M}}_{\text {ext } F, a} \supset$ $\mathcal{M}_{\text {int } F, a}$. This completes the proof.

The following example shows that in (3.14) the inclusion can be strict.

Example 3.1. Set $f(y)=\sqrt{|y|}$ if $|y| \leq 1$ and $f(y)=1$ if $|y|>1$. For $F: \mathbb{R}^{2} \rightarrow$ $\mathcal{B}\left(\mathbb{R}^{2}\right)$ given by

$$
F(x, y)=\left\{(u, v) \in \mathbb{R}^{2} \mid u \in[0,1], v \in[f(y), 2]\right\}, \quad(x, y) \in \mathbb{R}^{2},
$$

consider the following Cauchy problems:

$$
\begin{array}{lll}
\left(C_{\text {int } F, 0}\right) & (\dot{x}(t), \dot{y}(t)) \in \operatorname{int} F(x(t), y(t)), & (x(0), y(0))=(0,0), \\
\left(C_{\operatorname{ext} F, 0}\right) & (\dot{x}(t), \dot{y}(t)) \in \operatorname{ext} F(x(t), y(t)), & (x(0), y(0))=(0,0) .
\end{array}
$$

Put $I=[0,1 / 2]$. Clearly

$$
\operatorname{ext} F(x, y)=\{(0, f(y)),(0,2),(1, f(y)),(1,2)\}
$$

and thus $(0,0) \in \operatorname{ext} F(0,0)$, which shows that $\left(x_{0}(t), y_{0}(t)\right)=(0,0), t \in I$, is a solution of $\left(C_{\operatorname{ext} F, 0}\right)$. Let $(x(t), y(t)), t \in I$, be an arbitrary solution of $\left(C_{\text {int } F, 0}\right)$. As $x(0)=y(0)=0$ and $x(t)$ and $y(t)$ satisfy

$$
\dot{x}(t) \in(0,1), \quad \dot{y}(t) \in(f(y(t)), 2),
$$

it follows that $x(t)$ and $y(t)$ are strictly positive for every $t \in(0,1 / 2]$. Clearly $\dot{y}(t) \in(\sqrt{y(t)}, 2)$ for $t \in I$ a.e. since, by $(3.15), y(t) \leq 1$ for every $t \in I$. Let $0<\varepsilon<1 / 4$. Then for $t \in[\varepsilon, 1 / 2]$ a.e. we have $\dot{y}(t)>\sqrt{y(t)}$, and hence $\sqrt{y(t)}>$ $\sqrt{y(\varepsilon)}+\frac{t-\varepsilon}{2}, t \in[\varepsilon, 1 / 2]$, from which letting $\varepsilon \rightarrow 0$ one has

$$
y(t) \geq \frac{t^{2}}{4}, \quad t \in I .
$$

Consequently

$$
d\left(\left(x_{0}(.), y_{0}(.)\right), \mathcal{M}_{\operatorname{int} F, 0}\right) \geq \frac{1}{8},
$$

as $(x(t), y(t)), t \in I$, is an arbitrary solution of $\left(C_{\text {int } F, 0}\right)$. This shows that the inclusion $\overline{\mathcal{M}}_{\text {ext } F, 0} \supset \mathcal{M}_{\text {int } F, 0}$ is strict. 


\section{REFERENCES}

[1] J.P. Aubin, A. Cellina, Differential Inclusions, Springer-Verlag, Berlin, 1984. MR755330 (85j:49010)

[2] C. Castaing, M. Valadier, Convex Analysis and Measurable Multifunctions, Lecture Notes in Mathematics, Vol. 580, Springer-Verlag, Berlin, 1977. MR0467310 (57:7169)

[3] A. Cellina, "On the differential inclusion $\dot{x} \in[-1,1]$ ", Atti Accad. Naz. Lincei Rend. Cl. Sci. Fis. Mat. Natur. (8) 69 (1980), 1-6. MR641583 (83g:34012)

[4] A. Cellina, "A view on differential inclusions", Rend. Semin. Mat. Univ. Politec. Torino 63 (2005), 197-209. MR2201565 (2006i:34026)

[5] G. Choquet, Lectures on Analysis, Vols. I, II, III, Mathematics Lecture Notes Series, Benjamin, New York, Amsterdam, 1969. MR0250011 (40:3252), MR0250012 (40:3253), MR0250013 (40:3254)

[6] F.S. De Blasi, G. Pianigiani, "A Baire category approach to the existence of solutions of multivalued differential equations in Banach spaces", Funkcial. Ekvac. 25 (1982), 153-162. MR694909 (84e:34019)

[7] F.S. De Blasi, G. Pianigiani, "Differential inclusions in Banach spaces", J. Differential Equations 66 (1987), 208-229. MR871995 (88a:34096)

[8] F.S. De Blasi, G. Pianigiani, "Non-convex-valued differential inclusions in Banach spaces", J. Math. Anal. Appl. 157 (1991), 469-494. MR1112329 (92h:34037)

[9] F.S. De Blasi, G. Pianigiani, "Baire category and relaxation problems for locally Lipschitzian differential inclusions on finite and infinite time intervals", Nonlinear Analysis TMA, DOI 10.1016/j.na.2009.06.110

[10] T. Donchev, E. FARKhi and B.S. Mordukhovich, "Discrete approximations, relaxation, and optimization of one-sided Lipschitzian differential inclusions in Hilbert spaces", J. Differential Equations 243 (2007), 301-328. MR2371790 (2008j:49040)

[11] A.N. Godunov, "The Peano theorem in Banach spaces", Functional Anal. Appl. 9 (1975), 53-55. MR0364797 (51:1051)

[12] S. Hu, N.S. PAPAGEORGIOU, Handbook of multivalued analysis, Vols. I, II, Kluwer, Dordrecht, 1998. MR:1485775 (98k:47001)

[13] B.S. MoRDUKHOVICH, Variational analysis and generalized differentiation, Vols. I, II, Springer-Verlag, Berlin, 2006. MR2191744 (2007b:49003a) MR2191745 (2007b:49003b)

[14] N.S. PAPAgEORgIou, "On the bang-bang principle for nonlinear evolution inclusions", Aequationes Math. 45 (1993), 267-280. MR1212391 (94d:49010)

[15] G. Pianigiani, "Differential inclusions. The Baire category method", in Methods of nonconvex analysis, edited by A. Cellina, Lecture Notes in Math., 1446, Springer-Verlag, Berlin, 1990, 104-136. MR:1079761 (91k:49007)

[16] A. Plis, "Trajectories and quasitrajectories of an orientor field", Bull. Acad. Polon. Sci., Ser. Sci. Math. Astronom. Phys. 11 (1963), 369-370. MR0155072 (27:5014)

[17] A.A. Tolstonogov, Differential inclusions in a Banach space, Kluwer, Dordrecht, 2000. MR.1888331 (2003g:34129)

[18] A.A. Tolstonogov, "The bang-bang principle for controlled systems of subdifferential type", Proc. Steklov Inst. Math. (2005), Dynamical Systems and Control Problems, 222-233. MR2157141 (2006f:49007)

Dipartimento di Matematica, Università di Roma "Tor Vergata", Via della Ricerca SCientifica 1, 00133 Roma, Italy

E-mail address: deblasi@mat.uniroma2.it

Dipartimento di Matematica per le Decisioni, Università di Firenze, Via Lombroso 6/17, 50134 Firenze, Italy

E-mail address: giulio.pianigiani@unifi.it 